\title{
Supersonic kinks in Coulomb lattices
}

Juan F.R. Archilla ${ }^{1}$, Yuriy A. Kosevich ${ }^{2}$, Noé Jiménez ${ }^{3}$, Víctor J. Sánchez-Morcillo ${ }^{3}$, and Luís M. García-Raffi ${ }^{4}$

1 Grupo de Física No Lineal. Universidad de Sevilla. Departamento de Física, Aplicada I. ETSI Informática, Avda. Reina Mercedes, s/n. 41012-Sevilla (Spain)

2 Semenov Institute of Chemical Physics, Russian Academy of Sciences, ul. Kosygina 4, 119991 Moscow, Russia

3 Instituto de Investigación para la Gestión, Integrada de las Zonas Costeras, Universidad Politécnica de Valencia, c/ Paranimfo 1, 46730 Grao de Gandia (Spain)

4 Instituto de Matemática Pura y Aplicada, Universidad Politécnica de Valencia, Camino de Vera s/n, 46022 Valencia (Spain)

Summary. There exist in nature examples of lattices of elements for which the interaction is repulsive, the elements are kept in place because different reasons, as border conditions, geometry (e.g., circular) and, certainly, the interaction with other elements in the system, which provides an external potential. A primer example are layered silicates as mica muscovite, where the potassium ions form a two dimensional lattice between silicate layers. We propose an extremely simplified model of this layer in order to isolate the properties of a repulsive lattice and study them. We find that they are extremely well suited for the propagation of supersonic kinks and multikinks. Theoretically, they may have as much energy and travel as fast as desired. This striking results suggest that the properties of repulsive lattices may be related with some yet not fully explained direct and indirect observations of lattice excitations in muscovite.

Keywords: discrete excitations; repulsive lattices; intrinsic localized modes PACS: 63.20.Pw, 63.20.Ry, 63.50.+x, 66.90.+r, 82.20.-w

\section{Introduction}

Layered silicates are made up from two basic building blocks: a sheet of edgesharing $\left[\mathrm{SiO}_{4}\right]$ units, the tetrahedral sheet, and another one of edge-sharing $\left[\mathrm{MO}_{6}\right]$ units, the octahedral sheet. Muscovite is a mica in which a charged layer results from the isomorphic substitution of silicon by aluminium in the tetrahedral sheet [1]. The structure of muscovite is depicted at the left of Fig. 1. The figure at the right shows an illustration of the interlayer space of muscovite. It can be observed that both surfaces of the upper and lower tetrahedral sheets are formed by the basal oxygen atoms from the $\left[\mathrm{SiO}_{4}\right]$ tetrahedra, 

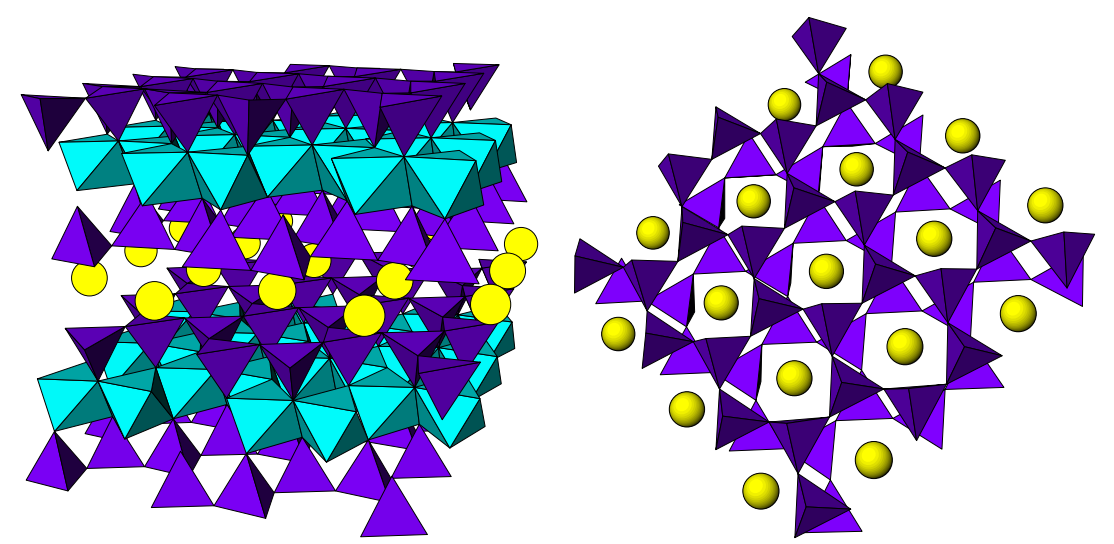

Fig. 1. Crystal unit cell of muscovite ICSD 34406. The circles represent the potassium ions forming the interlayer sheet. $(\mathrm{a}=5.19 \AA ; \mathrm{b}=9.02 \AA ; \mathrm{c}=20.0 \AA$; $\left.\beta=95.7^{\circ}\right)$

which form a rough hexagonal honeycomb structure. The interlayer balancing sheet is therefore sandwiched in between, potassium occupying the dimples left at the center of each pair of hexagonal cells. Each potassium ion is surrounded by six other potassium ions in the interlayer sheet with a ditrigonal symmetry.

Essentially, in both clay minerals and mica, $\left[\mathrm{SiO}_{4}\right]$ tetrahedra are linked into infinite two-dimensional networks by sharing three oxygens.

Mica muscovite has a very special characteristic, its recording capability. Tracks from particles as muons can be distinguished in the complex decoration, but the only explanation to most of the tracks is the presence of localized excitations, called quodons [2]. They propagate in the cation layer without mass transport along the lattice directions. Another interesting effect probably related with the existence of fast travelling localized perturbations was reported in Ref. [3]: experiments show that an alpha particle hitting one boundary of a muscovite layer provokes the ejection of an atom at the other side of the layer, several millimeters far from the excitation.

What makes the two dimensional, hexagonal lattice that form the cation layer so special? Transversal breathers related with the kinetics $[4,5,6]$ have been found in the cation layer, they have small energies below a few $\mathrm{eV}$, move slowly and radiating, therefore they are not good candidates for quodons. We advance the hypothesis that one reason could be that the ion lattice is made out of repulsive cations, $\mathrm{K}^{+}$ions in muscovite. As a first step to explore the implications of the hypothesis we study a one-dimensional model of potassium ions coupled through Coulomb potentials, and observe that supersonic kinks [7] with a very different range of energies can propagate long distances riding a sea of phonons and rebounding with others. 
It is certainly probable that a straightforward $2 \mathrm{D}$ generalization of the one dimensional model will not support one dimensional kinks. On the other hand, if the tracks in mica correspond to lattice excitations as we believe, they are quasi one-dimensional. Why they do not spread laterally is a key question that we are not prepared to answer at this stage but which is one of our most important midterm objectives. For that we will most likely have to introduce more characteristics of real mica and our goal is to find exactly which ones. But we think that understanding first what is happening in one dimension is a necessary first step as have been done so often and successfully in solid state physics.

Certainly, repulsive Coulomb lattices are not limited to the potassium sheet in mica. Electrons form a Wigner crystal [8] which have been extensively studied in several dimensions. See for example Ref. [9] on one dimensional crystal and Ref [10] for the first experimental 2D realization on a liquidhelium surface. Macroscopic repulsive Coulomb systems can be constructed in one dimension with magnets located with opposite poles in front of each other, although in this case friction is also present. This system has already been constructed by the authors and research on it is planned.

The outline of this article is as follows. In section 2 we describe the model and its justification, in section 3 we obtain the dispersion curves for the linearized system and compare them with the data for mica muscovite. Section 4 describes the tail analysis and their implication for possible travelling localized solutions. In section 5 the velocities of the kinks are obtained theoretically, while section 6 describes the results of numerical simulations and compare with the theory. After some comments about the effect of long range interaction the article finishes with the conclusions.

\section{Model equations}

We simplify the model of the potassium ions inside the layer of muscovite making the following assumptions: a) We consider the motions only along a lattice direction where the $K^{+}$ions have the shortest distance $a=5.19 \AA$, which becomes the lattice unit; b) We substitute the electric field created by the silicate layers by a uniform field, which is taken into account only conceptually; c) We consider only Coulomb interaction and only between nearest neighbors. This is justified because the negative charge above and below the $K^{+}$ions is rapidly screened by the surrounding negative charge. d) The effect of the surrounding negative charge is to keep the ions in place. This is incorporated into the system through fixed particles at the ends, so as the interatomic distance is the one observed in real muscovite. In Fig. 2 we show a sketch of the model.

Note, that however important the periodic potential in real mica might be, the existence of a the two-dimensional $K^{+}$does not need it. It comes about for the need to compensate the excess negative charge of the silicate 
layers. The latter determines also the surface charge density of the $K^{+}$layer, and, thus, the interatomic spacing. Repulsion of the ions determines a closed packed hexagonal structure.

With these assumptions the equation of motion of a $K^{+}$ion is given by:

$$
m \ddot{x}_{n}=-\frac{K_{c} \mathrm{e}^{2}}{\left(x_{n+1}-x_{n}\right)^{2}}+\frac{\mathrm{K}_{\mathrm{C}} \mathrm{e}^{2}}{\left(x_{n}-x_{n-1}\right)^{2}} .
$$

These coupled equations can be made dimensionless, by choosing the unit of distance, $a=5.19 \AA$, time, $\tau=\sqrt{m_{K} a^{3} / \mathrm{K}_{\mathrm{C}} e^{3}}=0.1989 \mathrm{ps} \simeq 0.2 \mathrm{ps}$, mass, $m_{K^{+}}=39.1 \mathrm{amu}$, and also defining the displacement with respect to the equilibrium position $u_{n}$, measured in lattice units as $x_{n}=a n+a u_{n}$. We get:

$$
\ddot{u}_{n}=-\frac{1}{\left(1+u_{n+1}-u_{n}\right)^{2}}+\frac{1}{\left(1+u_{n}-u_{n-1}\right)^{2}} .
$$

The system of $N$ atoms is kept in place (the chain is stable at equilibrium) by setting two extra fixed particles at positions $n=0$ and $n=N+1$. Therefore, $x_{0}$ and $x_{N+1}$ are always zero. The equations for the particles $\mathrm{n}=1$ and $\mathrm{n}=\mathrm{N}$, become

$$
\begin{aligned}
\ddot{u}_{1} & =-\frac{1}{\left(1+u_{2}-u_{1}\right)^{2}}+\frac{1}{\left(1+u_{1}\right)^{2}} \\
\ddot{u}_{N} & =-\frac{1}{\left(1-u_{N}\right)^{2}}+\frac{1}{\left(1+u_{N}-u_{N-1}\right)^{2}}
\end{aligned}
$$

These boundary conditions have been chosen for convenience, because with periodic boundary conditions two kinks are produced travelling with opposite directions and eventually colliding. In this article we are not trying to model border effects and far away from the borders the properties of phonons and kinks with different boundary condition are virtually identical in the simulations. As our physical model consists of a large number of atoms, periodic conditions are more convenient for the theory, but fixed ends are preferably for the numerical calculations. Somehow, fixed end are more realistic to model the interaction of a swift particle with the lattice border, but certainly the boundary potential has to be carefully chosen, which is also one midterm objective of the authors' research.

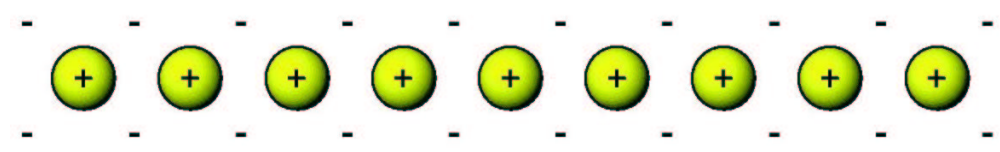

Fig. 2. Sketch of the Coulomb's chain. The yellow spheres represent the $K^{+}$ions surrounded by the negative charge of the silicate layers above and below. 


\section{Phonons}

For small amplitudes, the power-law potentials can be expanded in series, using that $1 /(1+y)^{2} \simeq 1-2 y+3 y^{2}-4 y^{3} \ldots$. Retaining cubic terms and smaller, Eq. (2) is reduced to

$$
\begin{aligned}
\ddot{u}_{n}= & 2\left(u_{n+1}+u_{n-1}-2 u_{n}\right)-3\left(u_{n+1}-u_{n}\right)^{2}+3\left(u_{n}-u_{n-1}\right)^{2} \\
+ & 4\left(u_{n+1}-u_{n}\right)^{3}-4\left(u_{n}-u_{n-1}\right)^{3}+\ldots .
\end{aligned}
$$

which is the $\alpha-\beta$ FPU equation. Neglecting nonlinear terms, it reduces to the discrete linear wave equation,

$$
\ddot{u}_{n}=\mathrm{c}^{2}\left(u_{n+1}+u_{n-1}-2 u_{n}\right)
$$

where $\mathrm{c}=\sqrt{2}$ will be shown to be the speed of sound. Note that, although it can be scaled out, we keep here the value of $\mathrm{c}$ in the equations. This makes easier the comparison with other results in the literature (different scalings are often used), and mainly with the standard scaling corresponding to $\mathrm{c}=1$. Also note that this linearized equation is attractive, i.e., if the distance $u_{n+1}-u_{n}$ increases the force on the particle $u_{n}$ is positive. The reason for it is the decreasing nature of the Coulomb force: when the distance with the particle in front increases the repulsion weakens and the repulsion from the particle behind becomes larger pushing the particle $n$ forward .

A phonon corresponds to small amplitude particle displacements of the form $u_{n}=\exp (\mathrm{i}(q n-\omega t)$, with $q$ and $\omega$ being the phonon wave number and frequency, respectively. By substitution in Eq. (5) we obtain the phonon dispersion equation:

$$
\omega=\omega_{M} \sin (q / 2) \quad \text { with } \quad \omega_{M}=2 \mathrm{c}, \quad \text { and } \quad q=\frac{2 \pi m}{N}, \quad m=0,1, \ldots N .
$$

$\omega_{M}=2 \mathrm{c}$ is the maximum frequency, corresponding to the mode $q=\pi$. The phase velocity $V_{p h}=\omega / q$ and the group velocity $V_{g}=\partial \omega / \partial q$ are given by

$$
V_{p h}=\mathrm{c} \frac{\sin (q / 2)}{q / 2} \quad, \quad V_{g}=\mathrm{c} \cos (q / 2)
$$

The speed of sound $c$ is recovered in the long wavelength limit, i.e., $\lim _{q \rightarrow 0} V_{p h}=$ $\lim _{q \rightarrow 0} V_{g}=\mathrm{c}$, as anticipated. This is also the maximum of both the phase and group velocities. The latter becomes zero for the boundary mode $q=\pi$.

The above are standard calculations, we include them for completeness but specially to compare the phonon properties in our model with actual experimental measurements in real samples. In Fig. 3 we plot the dispersion curve as obtained for our model in physical units (left), and compare it with data obtained from neutron spectroscopy and molecular dynamics in Ref. [11] (right). We can see that there is a general agreement in the shape and values. Similar curves have been also obtained in Refs. [13, 14]. 

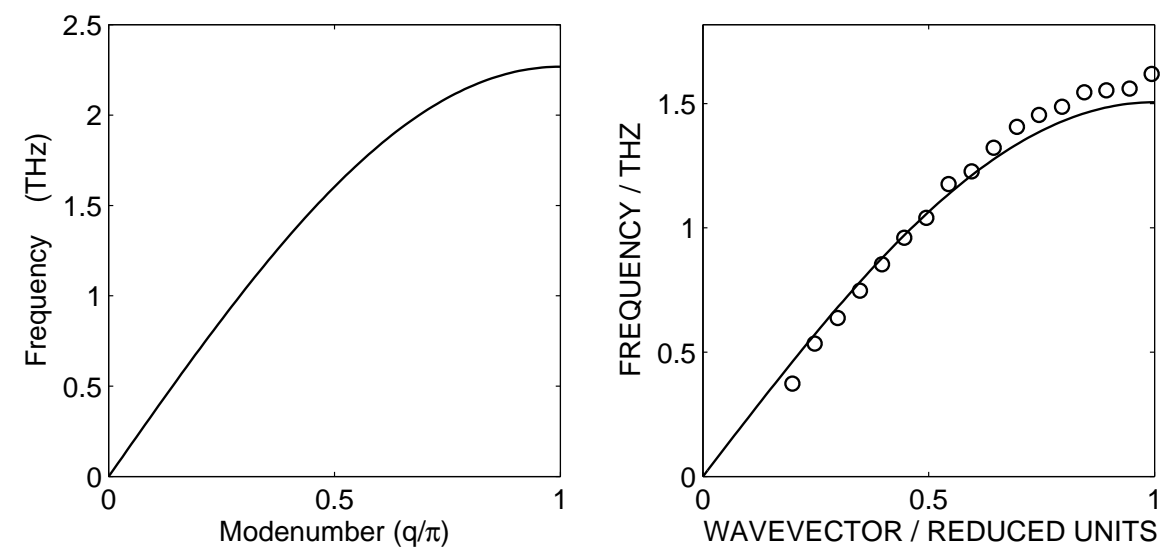

Fig. 3. Phonon dispersion curves obtained in the Coulomb's chain (right) and reproduced from Ref. [11] (left).

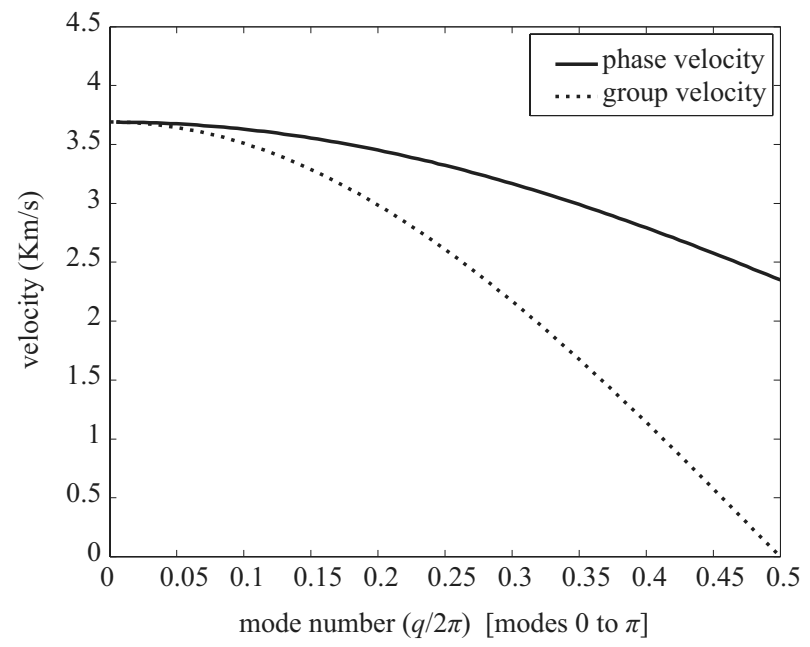

Fig. 4. Phase and group velocities in the Coulomb's chain, both give at $q \rightarrow 0$ the sound velocity, which can be compared with the values of $3.4-3.7 \mathrm{~km} / \mathrm{s}$ obtained in Ref. [12].

Figure 4 shows the phase and group velocities in physical units. Note that the predicted speed of sound is similar to that found in Ref. [12] of 3.4-3.7 $\mathrm{km} / \mathrm{s}$.

Since we are using a very simplified model, it may result surprising that it gives values similar to much more complex models and experimental data. The reason is that in our systems there are many real things: the atomic 
mass, the Coulomb interaction, and some external potentials that keep the chain at equilibrium at the real interatomic distance. This also supports our assumption that at least for small perturbations the dominant interaction is the Coulombian one.

\section{Localized travelling solutions}

In this section we consider different types of localized solutions supported by the Coulomb lattice. Although these solutions are large enough to be intrinsically nonlinear, they also have tails of small amplitude which abide the linear equation. Tails cannot exist by themselves but depends on the existence of the nonlinear solution, however they also exist and its study gives information about the parent excitation. We obtain first the tail properties and later we consider the fully nonlinear solutions, kinks and solitons. Tails are obtained with the linear system, therefore their properties are common to any system with the same linearization. However, they only give the conditions that the tails have to fulfill. The properties of existence of the nonlinear excitation, its velocity, amplitude and other characteristics have to be worked out with the whole nonlinear system.

\subsection{Tail analysis}

Consider a nonlinear localized moving excitation, travelling with velocity $V$. We also assume that its amplitude decays exponentially far away enough from the center. Tails are well described by the expression

$$
u_{n}=\exp (-\xi(n-V t)) \exp (\mathrm{i}(q n-\omega t)),
$$

which is valid for $n>V t$ if $\xi>0$ corresponding to the front tail of the structure and for $n<V t$ if $\xi<0$ corresponding to the back tail. We call $|\xi|$ the localization parameter and its inverse $\Lambda=1 /|\xi|$ the decay length. The limit case $\xi=0$ corresponds to extended phonons. Since the tail amplitude is by definition small, it satisfies the linear equation Eq. (5). Using that $u_{n+1}=$ $\exp (-\xi+\mathrm{i} q) u_{n}, u_{n-1}=\exp (\xi-\mathrm{i} q) u_{n}$ and $\dot{u}_{n}=(\xi v-\mathrm{i} \omega) u_{n}$, substitution of $u_{n}$ yields the following two equations for the real and imaginary part of Eq. (5):

$$
\xi^{2} V^{2}-\omega^{2}=2 \mathrm{c}^{2}(\cosh (\xi) \cos (q)-1) \quad ; \quad \xi V \omega=\mathrm{c}^{2} \sinh (\xi) \sin (q) .
$$

As there are two equation with four unknowns, we can express two of them as $\omega$ and $V$ as functions of the other two $\xi$ and $q$. First, the frequency $\omega$ is given by:

$$
\omega=\cosh (\xi / 2) \omega_{M} \sin (q / 2)=\cosh (\xi / 2) \omega_{\text {phonons }},
$$


with $\omega_{M}=2$ c. Note that Eq. (10) reduces to the phonon dispersion equation when there is no localization, i.e. $\xi \rightarrow 0$.

The velocity of the tail is given by:

$$
V=\frac{\sinh (\xi / 2)}{\xi / 2} \mathrm{c} \cos (q / 2)=\frac{\sinh (\xi / 2)}{\xi / 2} V_{\mathrm{g}, \text { phonons }} .
$$

Note that both $\sinh (\xi / 2) /(\xi / 2)$ and $\cosh (\xi / 2)$ are larger than the unity and also monotonically increasing functions in $|\xi|$, which means that the tails oscillate and move faster than the phonons. Except for the degenerate case $\xi=0$, which corresponds to no localization and no tail, i.e., to phonons.

\subsection{Oscillating tails}

Breathers are localized oscillating solutions with a frequency outside the phonon band, but there are neither stationary nor moving breathers in this system, a fact known for repulsive FPU systems [15]. However, the tail analysis can be applied to oscillating tails following or preceding a soliton or kink, which do exist in this system.

Travelling tails correspond to localized, vibrating, travelling excitations, that is $\xi, V$ and $\omega$ are all different from zero. From Eq. (10) we conclude that the tail frequency $\omega$ increases with the localization $\xi$. However, being inside the phonon band, i.e., $0<\omega<\omega_{M}=2$ c, the interaction with the phonons would produce the tail instability. The mode $q=\pi$ would be the most stable, as its frequency is always larger than $\omega_{M}$, but its velocity is zero. However, increasing the localization $\xi$ we can increase both the frequency and the velocity. However, values of $\xi$ larger than a few units are not realistic, neither values of the frequency of many times the maximum phonon frequency. So a kind of balance is needed. One notable particular case, introduced in Ref. [7], allows analytical estimations (see next section). It corresponds to solutions with $q=2 \pi / 3$, known as the magic number. For this case we obtain

$$
\omega=\sqrt{3} \mathrm{c} \cosh (\xi / 2) \quad ; \quad V=\frac{\mathrm{c} \sinh (\xi / 2)}{\xi} .
$$

Some estimations can be done. If $\omega>2$ c then $\xi>\xi_{0}=1.0986$, for $\xi=\xi_{0}$ each atom amplitude is exactly a third smaller than the previous one. For $\xi=\xi_{0}$, the oscillating tail velocity is $V \approx \mathrm{c} / 3$ or one third of the sound velocity. We need values of about $\xi=6.5$ to approach the sound velocity, but at such strong localization value the frequency is 11 times the maximum phonon frequency and a particle has an amplitude one thousand smaller than the previous one. Since such conditions are unrealistic, we can, in principle, consider that oscillating tails are subsonic. As we will see below, our simulations show that the most common behavior is an oscillating tail that is left behind the supersonic soliton bringing about a kink. 


\subsection{Supersonic solitons and kinks}

Solitons are localized, travelling non oscillatory excitations, that is with $V \neq 0$ and $\omega=0$. From Eq. (10) it follows that $\sin (q / 2)=0$ or $q=0$ (solitons are in-phase modes). Substituting into Eq. (11) we obtain the soliton velocity as a function of the localization $\xi$ :

$$
V_{\mathrm{sol}}=\frac{\sinh (\xi / 2)}{\xi / 2} \mathrm{c} .
$$

Two important conclusions arise: a) Solitons do not have an internal structure, as they come from the mode $q=0$ all the atoms are in-phase, and, the minimum value of the velocity of a soliton is $c$ the sound velocity, which is also the maximum phonon velocity. Therefore, the solitons, differently from oscillating tails, are supersonic.

As commented above it is possible to have different tail solutions for the front and back. For example, in principle, it is possible a front soliton, followed by a simple translation $x_{n}=a$, that would be a kink which leaves behind the atoms displaced one lattice unit, or the back tail may be an oscillatory tail, travelling at subsonic speed, being left behind by the supersonic soliton at the front as shown in Fig. 5. The front tail needs to be so steep to move in front of the soliton that it in practice means that the front of the soliton finishes abruptly, and the soliton becomes a kink. Of course, if these properties hold requires the study of the full system with the full potentials, which will be done in the next sections, but it is also essential to know the behavior of the system at low amplitudes, because part of the system correspond to that description when there is a localized nonlinear solution.

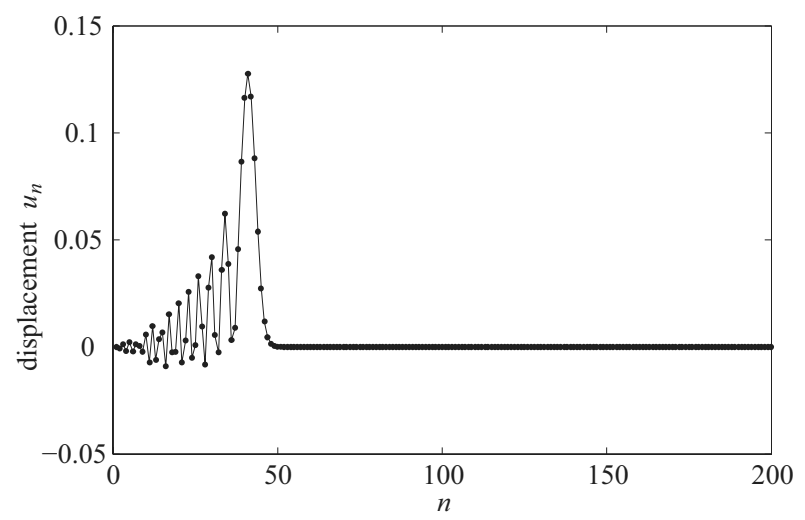

Fig. 5. Profile of the transitory state of a soliton, the subsonic oscillatory back tail will be left behind the supersonic soliton. The front tail becomes so steep that, in practice, it becomes an abrupt end of the perturbation. Therefore, the soliton will transform to a kink and the back tail without the support of the soliton will be dispersed in phonons. Dimensionless units. 


\section{Kink characteristics within the rotating wave approximation. Magic wave number.}

The previous tail analysis was derived from the linear equation (5). Is thus generic for many systems of coupled oscillators, although the specific values in physical units are specific for our system, as the phonon frequencies and speeds. We can obtain properties of the full nonlinear system using the Rotating Wave Approximation (RWA) [16], which consists in an approximation of the Fourier series up to the first harmonic.

It is convenient to define a new variable, the relative displacement or strain of the chain, as $v_{n}=u_{n}-u_{n-1}$. From Eq. (3), we can easily obtain the evolution equation for the strain as

$$
\ddot{v}_{n}=2 F_{n}-F_{n+1}-F_{n-1} ; \quad \text { with } \quad F_{n}=\frac{1}{\left(1+v_{n}\right)^{2}} .
$$

Kinks are characterized by a very steep profile, with only a few particles moving at a given time. A solution presenting this behavior is given (in the strain representation) by a half-wavelength structure with the magic wave number $q=2 \pi / 3$. Following Ref. [7], we propose the following ansatz, describing a kink travelling to the right

$$
v_{n}=-\frac{A}{2}(1+\cos (q n-\omega t)) \quad \text { if } \quad-\pi<q n-\omega t<\pi
$$

and $v_{n}=0$ otherwise. Eq. (15) represents a solution where 3 particles (the kink core) are in motion, while the others remain at rest. Note that this ansatz is only an approximation, although very good for intermediate to large amplitudes as it will be shown below.

When we substitute (15) in the equation of motion (14), nonlinearity generates multiple frequencies. The RWA approximation states that nonlinear contributions higher than second harmonics can be neglected. This implies that the force terms can be approximated by their first-order Fourier expansions, as $F_{n}=a_{0}+a_{1} \cos (q n-\omega t)$. On the other hand, the left high side of Eq. (14) gives, after substitution of Eq. (15), $\ddot{v}_{n}=(A / 2) \omega^{2} \cos (q n-\omega t)$ which is equal to the first harmonic term of the right hand side. The zero-harmonic cancels out due to the dependency of the rhs on $F_{n}$. The first harmonic of $F_{n}$ is given by:

$$
a_{1}=\frac{1}{\pi} \int_{-\pi}^{\pi} \frac{\cos (\theta) \mathrm{d} \theta}{\left(1-\frac{A}{2}-\frac{A}{2} \cos (\theta)\right)^{2}}
$$

For $A<1$ we obtain

$$
a_{1}=\frac{A}{(1-A)^{3 / 2}} .
$$

Therefore, substituting $\ddot{v}_{n}$ and $F_{n}=a_{0}+a_{1} \cos (q n-\omega t)$ in Eq. (14), is straightforward to obtain the frequency and velocity, as functions of the amplitude A 


$$
\omega=\frac{1}{(1-A)^{3 / 4}} \omega_{M} \sin (q / 2) ; \quad V=\frac{\omega}{q}=\frac{1}{(1-A)^{3 / 4}} \mathrm{c} \frac{\sin (q / 2)}{q / 2}
$$

These magnitudes are equal to the corresponding ones for phonons, multiplied by a factor $1 /(1-A)^{3 / 4}$, which of course tend to the unity when $A$ is small, but that diverges when $A$ approaches to unity. We can see the kink velocities in Fig. 6.

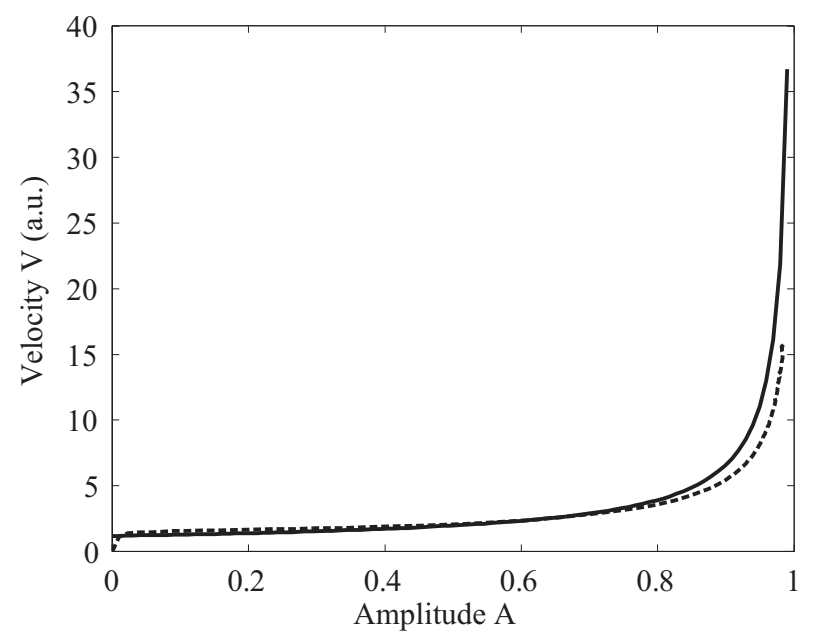

Fig. 6. Comparison of the numerical (dashed line) and theoretical (continuous line) velocities of kinks within the RWA, for the magic wave number $q=2 \pi / 3$. Dimensionless units.

\section{Numerical results}

We have done a systematic numerical study to determine the kink properties. The method to excite kinks is described in Ref. [7]. It consists in perturbing sinusoidally the first particle in the chain during half a period, with an amplitude $A_{0}$, so that the initial and final positions are both zero. Increasing the amplitude of the perturbation $A_{0}$ the velocity of the kink increases, and eventually, two, three, and any number of kinks are produced, travelling one after the other. The dependency of the velocity and number of kinks with the initial amplitude is shown in Fig. 7.

Fig. 8 shows the profile and the evolution of a single kink with a velocity twice the velocity of sound. Fig. 9 shows two kinks with velocities about three and five times the velocity of sound. 


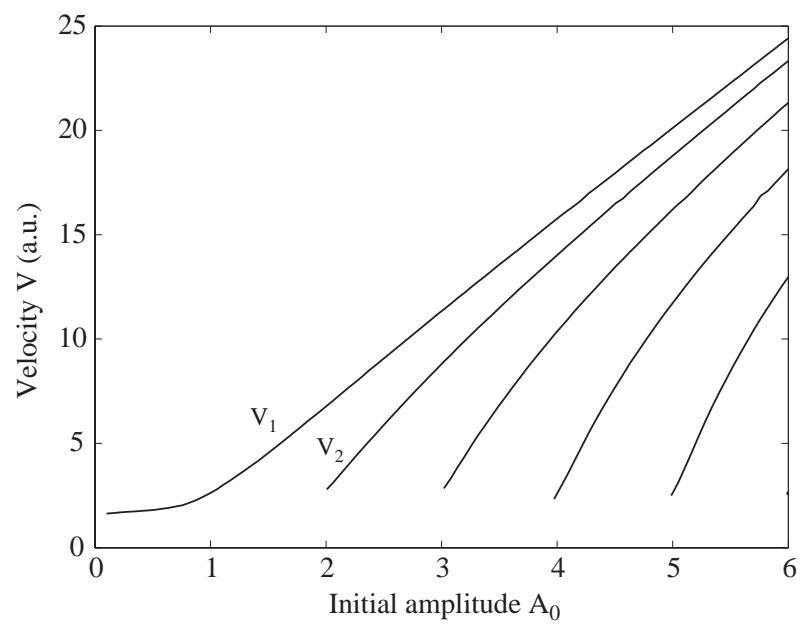

Fig. 7. Velocity and number of kinks as a function of the initial excitation amplitude $A_{0}$. Dimensionless units.

The kinks rebound at the borders and when they encounter each other. They are composed of particles moving in the direction of the kink and particles travelling in opposite directions cannot cross because that would need an infinite potential energy.

The velocity of the kinks $V$ increases with their amplitude $A$, tending to infinity with an asymptote at $A=1$. Note that $A=1$ would mean that the distance between particles $d_{n}=1+v_{n} \rightarrow 0$ which means an infinite energy. We have already commented the good agreement with the numerical results with the theory shown in Fig. 6 .

The relationship between the kink velocity with the decay length $\Lambda=1 / \xi$ given in Eq. (11) is shown in Fig. 10 compared with the numerical results, showing a perfect agreement.

In physical units, kinks can have any velocity and energy, for example we have found velocities up to $27 \mathrm{~km} / \mathrm{s}$ or eight times the sound velocity and energies from a few $\mathrm{eV}$ to hundreds of them. Whether these kinks exist and with which energies in mica muscovite remains and open question, but they seem a very good candidate.

\section{Effect of the interaction with several neighbors}

It is well know that for long-range interaction with a $1 / r$ potential the speed of sound diverges for a large system and therefore supersonic kinks cannot exist. Sound propagation is restored due to the screening of Coulomb's law by the electrons in a solid. It is not clear how many neighbors is reasonable 

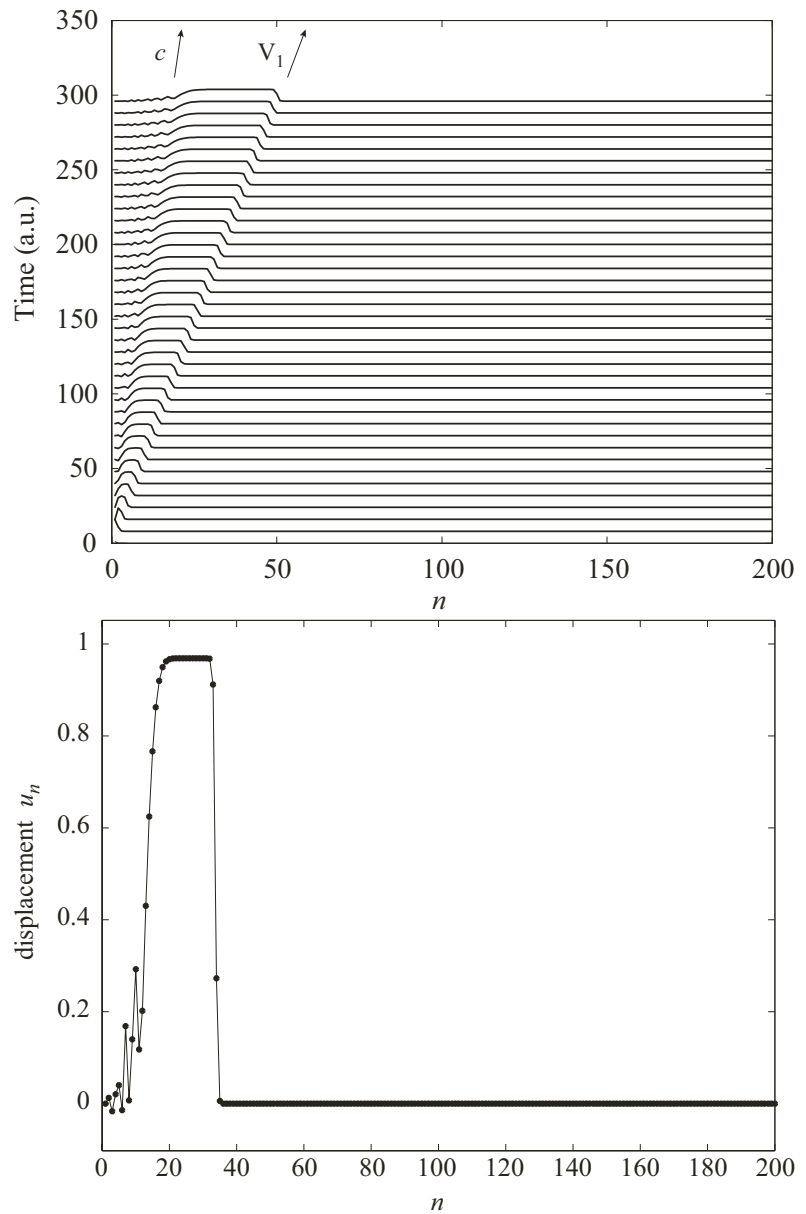

Fig. 8. Top: Profile and evolution of a single kink for $A_{0}=1.05$. Bottom: Detail of the displacements at $t=200$.

to take into account and we have made simulations in order to find out if kinks survive when longer range interaction is considered. We have found that when we increase the number of neighbors the kinks velocity diminishes and they become wider, with more than 3 particles forming them. For more than 10 neighbors we have not been able to produce kinks. When the study is completed, detailed results will be published elsewhere.

\section{Conclusions}

In minerals like mica muscovite there exist layers of repulsive ions all with the same charge sign forming a two-dimensional lattice. Muscovite shows the 

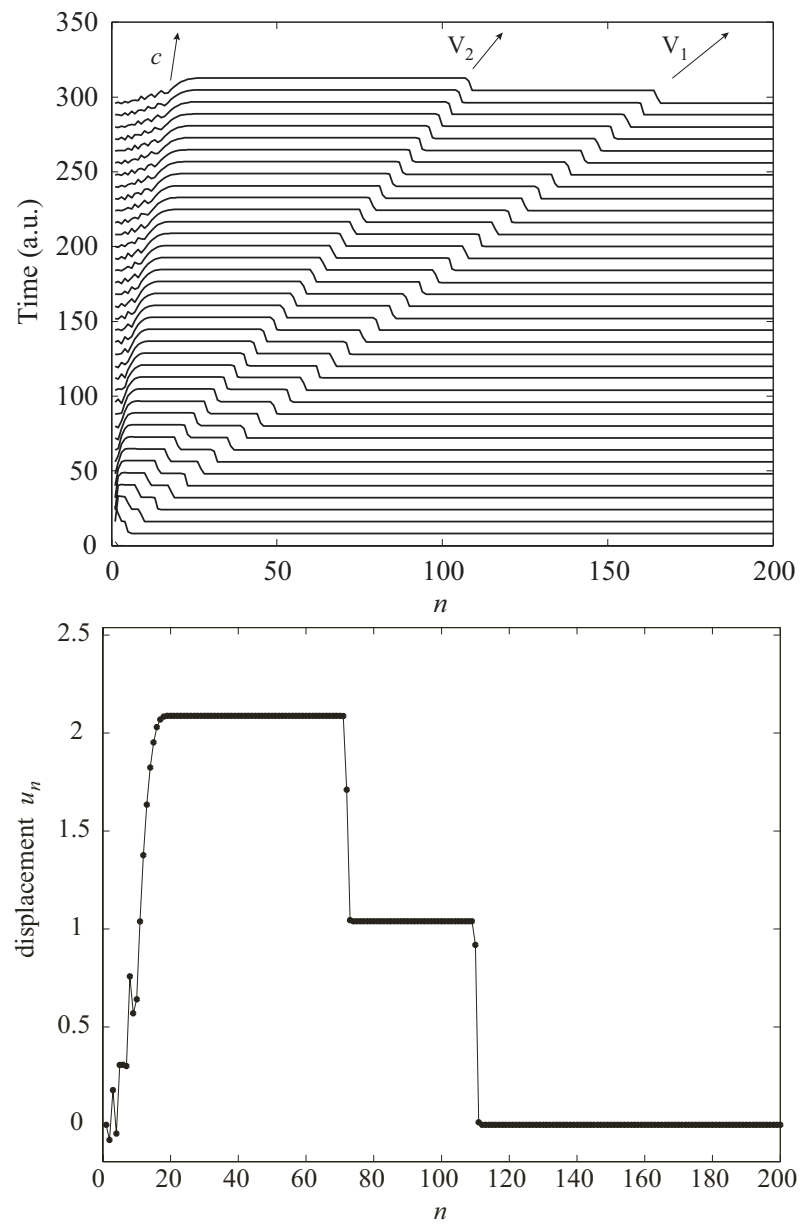

Fig. 9. (Top: excitation of multikinks for $A_{0}=2.5$. Bottom: Detail of the displacements at $t=200$ where two kinks are observed.

striking property of having been able to record the tracks of energetic particles as positrons and muons, but also shows tracks of what seem lattice localized excitations called quodons. Also an experiment seems not to have any other explanation. Motivated by the fact that an essential, but disregarded, characteristic of the $K^{+}$lattice is that all the particles repel each other, we study a minimal system made out of repulsive particles. We have found both theoretically and numerically that solitons in a transitory state and supersonic kinks and multikinks can travel very fast and thus are an efficient means of transporting localized energy along the lattice. The study of more realistic extensions of this model are underway and will be reported elsewhere. 


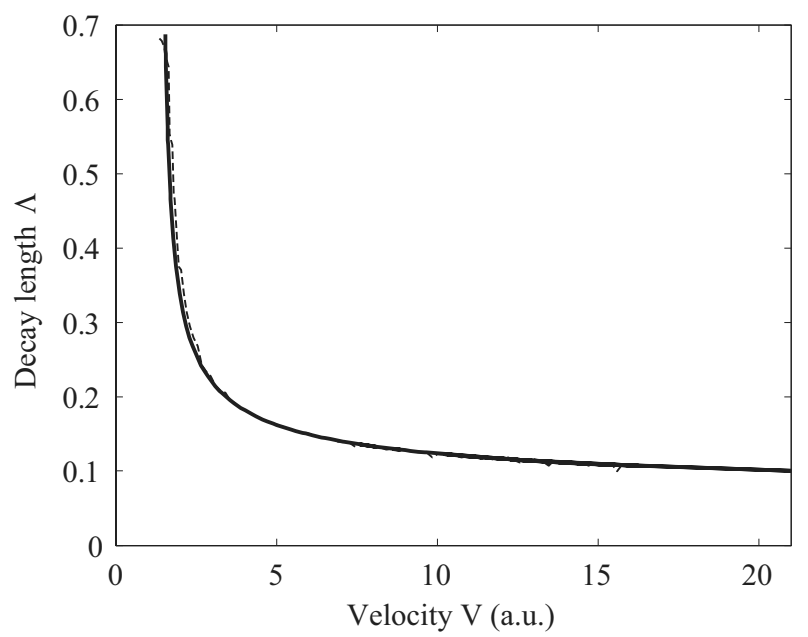

Fig. 10. Comparison of the numerical (dashed line) and theoretical (continuous line) decay lengths as a function of the velocity.

\section{Acknowledgments}

JFRA acknowledges financial support from the project FIS2008-04848. The work of VSM and LMGR was also supported by projects FIS2011-29731-C0202 and MAT2009-09438 respectively. All projects are granted by the Spanish Ministerio de Ciencia e Innovación. All authors acknowledges Mike Russell for continuous discussions.

\section{References}

1. L. A. Pérez-Maqueda, F. Franco, M. A. Avilés, J. Poyato and J. L. PérezMaqueda. Effect of sonication on particle-size distribution in natural muscovite and biotite. Clays and Clay Miner., 51 (2003) 701-708.

2. K. Kroneberger, M. Schosnig, F. M. Russell and K.O. Groeneveld. Search for solitons in solids. Rad. Meas, 23 (1994) 209-213.

3. F. M. Russell and J. C. Eilbeck. Evidence for moving breathers in a layered crystal insulator at 300K. Europhys. Lett., 78 (2007) 10004.

4. J. F. R. Archilla, J. Cuevas, M. D. Alba, M. Naranjo and J. M. Trillo. Discrete breathers for understanding reconstructive mineral processes at low temperatures. J. Phys. Chem. B, 110, 47 (2006) 24112-24120.

5. JFR Archilla, J Cuevas and F R Romero. Effect of breather existence on reconstructive transformations in mica muscovite. AIP Conf. Proc., 982, 1 (2008) $788-791$. 
6. V. I. Dubinko, P. A. Selyshchev and J. F. R. Archilla. Reaction-rate theory with account of the crystal anharmonicity. Phys. Rev. E, 83, 4 (2011) 13.

7. Yu. A. Kosevich, R. Khomeriki and S. Ruffo. Supersonic discrete kink-solitons and sinusoidal patterns with magic wave number in anharmonic lattices. Europhys. Lett., 66 (2004) 21-27.

8. E. Wigner. On the interaction of electrons in metals. Phys. Rev. Lett., 46 (1934) 1002-1011.

9. K. A. Matveev, A. V. Andreev and M. Pustilnik. Equilibration of a onedimensional wigner crystal. Phys. Rev. Lett., 105 (2010) 046401.

10. C. C. Grimes and G. Adams. Evidence for a liquid-to-crystal phase transition in a classical, two-dimensional sheet of electrons. Phys. Rev. Lett., 42 (1979) $795-798$.

11. D.R. Collins, W.G. Stirling, C.R.A. Catlow and G. Rowbotham. Determination of acoustic phonon dispersion curves in layer silicates by inelastic neutron scattering and computer simulation techniques. Phys. Chem. Miner., 19 (1993) $520-527$.

12. G. Brudeylins and D. Schmicker. Elastic and inelastic helium atom scattering at a cleaved mica sheet. Surf. Sci., 333 (1995) 237-242.

13. N Wada and WA Kamitakahara. Inelastic neutron and raman scattering studies of muscovite and vermiculite layered silicates. Phys. Rev. B, 43 (1991) 2391.

14. SL Chaplot and et al. Inelastic neutron scattering and lattice dynamics of minerals. Eur. J. Min., 14 (2002) 291.

15. James G., P. Kevrekidis and J. Cuevas. Breathers in oscillator chains with hertzian interactions. Submitted.

16. J B Page. Asymptotic solutions for localized vibrational modes in strongly anharmonic periodic systems. Phys. Rev. B, 41 (1990) 7835. 